\title{
Charge transfer between photosynthetic proteins and hematite in bio-hybrid photoelectrodes for solar water splitting cells
}

Greta Faccio ${ }^{1}$, Krisztina Gajda-Schrantz ${ }^{2,3,4}$, Julian Ihssen ${ }^{1}$, Florent Boudoire ${ }^{2,5}$, Yelin Hu ${ }^{2,6}$, Bongjin Simon Mun ${ }^{7}$, Debajeet K Bora ${ }^{2}$, Linda Thöny-Meyer ${ }^{1}$ and Artur Braun ${ }^{2^{*}}$

\begin{abstract}
Functionalization of the hematite photoanode with the photosynthetic light antenna protein C-phycocyanin (PC) can yield substantial enhancement of the photocurrent density. Photoelectrochemical cells with bio-hybrid electrodes from photosynthetic proteins and inorganic semiconductors have thus potential for the use in artificial photosynthesis. We investigate here processing routes for the functionalization of hematite photoanodes with PC, including in situ co-polymerization of PC with enzymatically-produced melanin, and using a recombinant PC genetically engineered to carry a hexa-histidine tag (aHisPC). First, the effect of the immobilisation of PC on the electrode morphology and photocurrent production is evaluated. Then, the electronic charge transfer in dark and light conditions is assessed with electrochemical impedance spectroscopy and valence band (VB) X-ray photoemission spectroscopy. The relative shift of the VB spectrum towards the Fermi energy $E_{F}$ upon illumination is smaller for the more complex processed coating and virtually disappears for aHisPC immobilised with a melanin film. Optimal conditions for protein immobilisation are determined and the dark currents benefit most from the most advanced protein coating processes.
\end{abstract}

Keywords: Artificial photosynthesis; Antenna protein C-phycocyanin; Hematite photoanode; Impedance spectroscopy; Valence band (VB) X-ray photoemission spectroscopy

\section{Background}

Hematite, a ferric oxide with $2.1 \mathrm{eV}$ optical band gap energy, is considered a low cost and environmentally benign photoanode material for solar hydrogen production by water splitting in photoelectrochemical cells (PEC). The research and development of photoelectrodes for PEC is focused on the tailoring of the electronic structure and the microstructure, and recently also on the design of hetero structures. The functionalization of a photoelectrode with light harvesting proteins was recently demonstrated for hematite using C-phycocyanin (PC) [1], which constitutes basically a dye sensitization.

Dye sensitization [2] is an established technology for the extension of the light absorption range of inorganic

\footnotetext{
* Correspondence: Artur.Braun@alumni.ethz.ch

${ }^{2}$ Laboratory for High Performance Ceramics, Empa, Swiss Federal Laboratories for Materials Science and Technology, Überlandstrasse 129, 8600 Dübendorf, Switzerland

Full list of author information is available at the end of the article
}

photoelectrodes for solar energy conversion. To be fully functional, the electric charge generated in the dye upon light absorption needs to be transferred to the photoelectrode, i.e. the interface between the dye and the photoelectrode must warrant proper charge transfer for electrons or holes.

We recently developed a process which enhances the photocurrent of hematite thin-film electrodes by immobilising light-harvesting proteins such as $\mathrm{PC}$ on the surface [1]. So far, the functionalization has been performed with the commercially available PC from Spirulina sp. which contains the $\alpha$ - and $\beta$-subunits of the protein. In the present study, we evaluate the simpler $\alpha$-subunit of PC from Synechocystis sp. PCC6803 which is recombinantly produced in E. coli and engineered to carry an $\mathrm{N}$-terminal hexa-histidine tag ( $\alpha$ HisPC) [3]. The electronic charge transfer is investigated by electrochemical impedance spectroscopy (EIS) and with valence band $\mathrm{X}$-ray photoemission spectroscopy (VB PES).

\section{实}




\section{Methods}

\subsection{Materials}

1,1'-Carbonyldiimidazol (CDI, >97\%), 1,4-dioxane (99.8\%), 2,2'-azino-bis(3-ethylbenzothiazoline-6-sulfonic acid) diammonium salt (ABTS, >98\%), monopotassium phosphate $\left(1 \mathrm{~mol} \mathrm{l}^{-1}\right)$, hydrogen dipotassium phosphate $\left(1 \mathrm{~mol} \mathrm{l}^{-1}\right)$, potassium hydroxide $(\mathrm{KOH}, 95 \%)$, iron nitrate nonahydrate (>98\%), tetrahydrofuran (THF, >99.9\%), sodium chloride $\left(\mathrm{NaCl}, 5 \mathrm{~mol} \mathrm{l}^{-1}\right)$, L-tyrosine (>99\%), Tris- $\mathrm{HCl}(>99 \%)$ and C-phycocyanin isolated from Spirulina sp. (99.9\%) were purchased from Sigma-Aldrich. Oleic acid (>60\%) and L (+)-tartaric acid (>99.5\%) were purchased from Fluka. Tyrosinase from Verrucomicrobium spinosum was produced in E. coli and proteolitically activated as described in [4].

\subsection{Production and purification of C-phycocyanin from Synechocystis sp. PCC6803}

The $\alpha$-subunit of PC from Synechocystis sp. PCC6803 was produced in an N-terminally His-tagged form according to [3] and purified with a modified protocol from [5]. Briefly, strain E. coli DH5 $\alpha$ was co-transformed with plasmids pAT101 and pBD414V and transformants were selected for resistance to both kanamycin $\left(50 \mu \mathrm{g} \mathrm{ml}^{-1}\right)$ and spectinomycin $\left(100 \mu \mathrm{g} \mathrm{ml}^{-1}\right)$. Cultures were routinely set up in TB medium (500 ml volume), incubated at $37^{\circ} \mathrm{C}$ under constant shaking (180 rpm), and expression was induced by adding IPTG (final conc. $1 \mathrm{mM}$ ). Cells were harvested by centrifugation $17 \mathrm{~h}$ post-induction and stored at $-20^{\circ} \mathrm{C}$. Upon thawing and re-suspension in $100 \mathrm{mM}$ potassium phosphate buffer with $\mathrm{pH} 7.5\left(5 \mathrm{ml} \mathrm{g}^{-1}\right.$ wet cell weight), lysozyme (final conc. $1 \mathrm{mg} \mathrm{ml}^{-1}$ ) and a protease inhibitor mix (Complete Protease Inhibitor Cocktail Tablets, Roche) were added. The cell suspension was then subjected to a freeze-thaw cycle at $-20^{\circ} \mathrm{C}$. Cells were further disrupted by sonication, and the cell-free extract, isolated by ultracentrifugation, was loaded onto a HisTrap column (1 ml, GE Healthcare Life Sciences) for purification by immobilized metal ion affinity chromatography (IMAC). $\alpha$ HisPC was eluted with imidazole (conc. $200 \mathrm{mM}$ ) in $100 \mathrm{mM}$ potassium phosphate ( $\mathrm{pH} 7.5)$. $\alpha$ HisPC-containing fractions were concentrated and desalted with a Vivaspin ultrafiltration device (10000 cut-off, Sartorius). The purified $\alpha$ HisPC was stored at $-20^{\circ} \mathrm{C}$ for further use. Protein concentrations of $\alpha$ HisPC solutions were determined spectrophotometrically with a Synergy Mx spectrophotometer (Biotek, Switzerland) and using an extinction coefficient of $140000 \mathrm{M}^{-1} \mathrm{~cm}^{-1}$ at $620 \mathrm{~nm}$. Protein purity was assessed spectrophotometrically as the ratio of the absorbance at $280 \mathrm{~nm}$ and at $620 \mathrm{~nm}$.

\subsection{Hematite films and single crystals}

Hematite films were prepared as described previously [6]. Fluorine doped tin oxide (FTO) coated glass slides $(12 \mathrm{~mm} \times 30 \mathrm{~mm} \times 2 \mathrm{~mm}$, TEC- 8 from Hartford Glass
Inc.) were automatically dip coated (DipMasterTM-50, Chemat Technology Inc., USA) with the precursor solution and dried at $75^{\circ} \mathrm{C}$ for $5 \mathrm{~min}$ on a hot plate. The samples were then further annealed for $30 \mathrm{~min}$ at $500^{\circ} \mathrm{C}$ in an air vented muffle furnace. Dip coating and annealing were repeated three times so as to obtain a film of four layers of hematite, which has typically a thickness of 500 to $600 \mathrm{~nm}$. Prevalence of the hematite phase was confirmed with X-ray diffraction (Siemens D5000).

The thickness of the hematite films was determined with a mechanical profilometer (XP-1 Stylus Profiler by Ambios Technology). The morphology of the pristine and protein coated hematite films was investigated with a Philips XL 30 scanning electron microscope (SEM, Oxford instruments).

Hematite single crystals with (0001) orientation were purchased from SurfaceNet GmbH, Rheine, Germany. They had a size of $5 \mathrm{~mm} \times 5 \mathrm{~mm} \times 1 \mathrm{~mm}$ size and were Epi surface polished.

\subsection{Coating of hematite with proteins and melanin}

Similarly to [1], the hematite thin films were first dipcoated with a $0.001 \%$ agarose solution, let dry, and second with $\sim 100 \mu \mathrm{cm}^{-2}$ of a $30 \mathrm{mg} \mathrm{ml}^{-1}$ CDI solution prepared in dioxan. Protein immobilisation was carried out by coating the hematite surface with a melanin-forming mixture containing PC or $\alpha$ HisPC (concentration $0.2 \mathrm{mg} \mathrm{ml}^{-1}$ ), L-tyrosine (concentration $0.1 \mathrm{mg} \mathrm{ml}^{-1}$ ), and tyrosinase (50 $\mathrm{g} \mathrm{m} \mathrm{m}^{-1}$ concentration) in $0.05 \mathrm{M}$ phosphate buffer saline (PBS; $160 \mathrm{~g} \mathrm{l}^{-1} \mathrm{NaCl}, 4 \mathrm{~g} \mathrm{l}^{-1} \mathrm{KCl}, 28.88 \mathrm{gl}^{-1} \mathrm{Na}_{2} \mathrm{HPO}_{4}$, $4.4 \mathrm{~g} \mathrm{l}^{-1} \mathrm{KH}_{2} \mathrm{PO}_{4}, \mathrm{pH}$ 7.2-7.4) for $3 \mathrm{~h}$. The surface was dried between all the steps, eventually rinsed with PBS and distilled water, and then stored at $4{ }^{\circ} \mathrm{C}$ in the dark until the actual measurements were performed. Because the tyrosinase preparation contains traces of trypsin, the protease inhibitor phenylmethanesulfonyl fluoride (PMSF) was added to the coating mixture at a $1 \mathrm{mM}$ final concentration. The thickness of the films treated with melanin alone, or with melanin plus PC or with melanin plus $\alpha$ HisPC was below $1000 \mathrm{~nm}$. All samples were prepared in either duplicate or triplicate and average values are reported throughout this paper.

In addition to the aforementioned thin hematite films on FTO-coated glass slides, three (0001) hematite single crystals were coated with:

1) Melanin plus $\alpha$ HisPC: $50 \mu \mathrm{l}$ solution containing $\alpha$ HisPC, L-tyrosine and tyrosinase in PBS (concentrations as above) after pre-treatment of the hematite single crystal with agarose and CDI solution (as described above).

2) PC only: $50 \mu \mathrm{l}$ solution containing PC, after pretreatment of the hematite single crystal with agarose and CDI solution (as described above).

3) Melanin plus PC: $50 \mu$ l solution containing PC and the melanin-forming mixture (as described above), after 
pre-treatment of the hematite single crystal with agarose and CDI solution (as described above).

\subsection{Photoelectrochemical assessment of the films}

For the assessment of the photoelectrochemical properties of the photoanode, we largely followed the recommendations provided in [7], when not otherwise stated in this manuscript. Linear voltammetry in the dark and under illumination was conducted using a specifically designed spectro-photoelectrochemical cell (known as EPFL cappuccino cell) made of polyether ether ketone (PEEK) and a potentiostat (Voltalab80 PGZ 402). The hematite photoanode was connected as the working electrode in a three electrode configuration. A platinum sheet of $5 \mathrm{~mm} \times 5 \mathrm{~mm}$ was used as counter electrode and an $\mathrm{Ag} / \mathrm{AgCl}$ (with saturated $\mathrm{KCl}$ ) electrode was used as reference electrode. The electrolyte was $0.05 \mathrm{M}$ PBS. Sunlight was simulated by a 1 Sun Oriel Lamp (L.O.T.Oriel AG) with a quartz filter, corresponding to AM 1.5 global standard solar spectrum. The bias potential was scanned from $0 \mathrm{mV}$ to $1000 \mathrm{mV}$. All shown results are the arithmetic average of data of at least three consecutive measurements. Electrochemical impedance spectra were recorded with the Voltalab80 PGZ 402 using the same cell in the frequency range from $100 \mathrm{kHz}$ to 100 $\mathrm{mHz}$ with 20 data points per decade and a perturbation amplitude of $10 \mathrm{mV}$. Spectra of hematite films coated on FTO glass and of variously prepared PC films on hematite were recorded. Dark and light conditions as described above were applied during the impedance measurements.

\subsection{Photoemission spectroscopy}

$\mathrm{X}$-ray photoemission (PES) spectra were recorded at beamline 8-1 at Stanford Synchrotron Radiation Lightsource, Menlo Park, CA. Specifically, the spectra were recorded under Fe 2p resonant condition with 53.2, 54.4 and $55.8 \mathrm{eV}$ photon energy, so that the valence band spectra were particularly representing the $\mathrm{Fe}$ relevant features. The PES spectra were measured from the surface finished (0001) hematite single crystals that were coated with PC in the aforementioned different processing routes. The measurements of PES were carried out under ultrahigh vacuum condition, i.e. $10^{-10}$ mbar base pressure. At the time of experiment, the films could be considered dry by ambient conditions. A 1.5 AM solar simulator (HAL-302 Solar Simulator, 350-750 nm, Asahi Spectra, Japan) was used to illuminate the samples during the PES experiments.

\section{Results and Discussion}

We have recently observed that the photocurrent density of a hematite electrode increases when the light-harvesting protein phycocyanin $(\mathrm{PC})$ is copolymerized with melanin on its surface. In the present work we investigate the influence of PC concentration, the time of incubation with the melanin-forming mixture on the photocurrent. In addition to the commercially available PC preparation, we test the effect of a recombinantly produced form of $\mathrm{PC}$ on the performance of the photoelectrochemical cell (PEC). Each sample is measured prior to and after the immobilization of proteins under pH-neutral conditions in PBS, if not mentioned otherwise.

\subsection{Production of recombinant aHisPC}

The $\alpha$-subunit of PC from Synechocystis sp. PCC6803 is produced in recombinant form in $E$. coli with a twoplasmid system [3]. Carrying an N-terminal Hexahistidine tag, $\alpha$ HisPC is purified by immobilized metal ion affinity chromatography (IMAC). The solution containing the purified $\alpha$ HisPC has a vivid blue colour. Differently from $\mathrm{PC}$ that has a maximum absorbance peak at $620 \mathrm{~nm}$, the purified $\alpha$ HisPC shows maximum optical absorbance at $626 \mathrm{~nm}$ (Figure 1).

Glazer et al. reported that the peak of maximum absorbance of C-phycocyanin when composed of both $\alpha$ - and $\beta$ subunits results from the sum of the absorbance peaks of both subunits. The $\alpha$-subunit absorbs at a longer wavelength than the $\beta$-subunit, e.g. the $\alpha$-subunit has an absorbance maximum at $620 \mathrm{~nm}$ and the $\beta$-subunit at $608 \mathrm{~nm}$ in C-phycocyanin from Synechococcus sp. [9].

Similarly to what has been reported for PC [8], incubation under alkaline conditions, e.g. in the presence of $1 \mathrm{M} \mathrm{KOH}$, absorbance in the $600-620 \mathrm{~nm}$ region is rapidly annulled and a novel absorbance peak at $750 \mathrm{~nm}$ is detected (Figure 1).

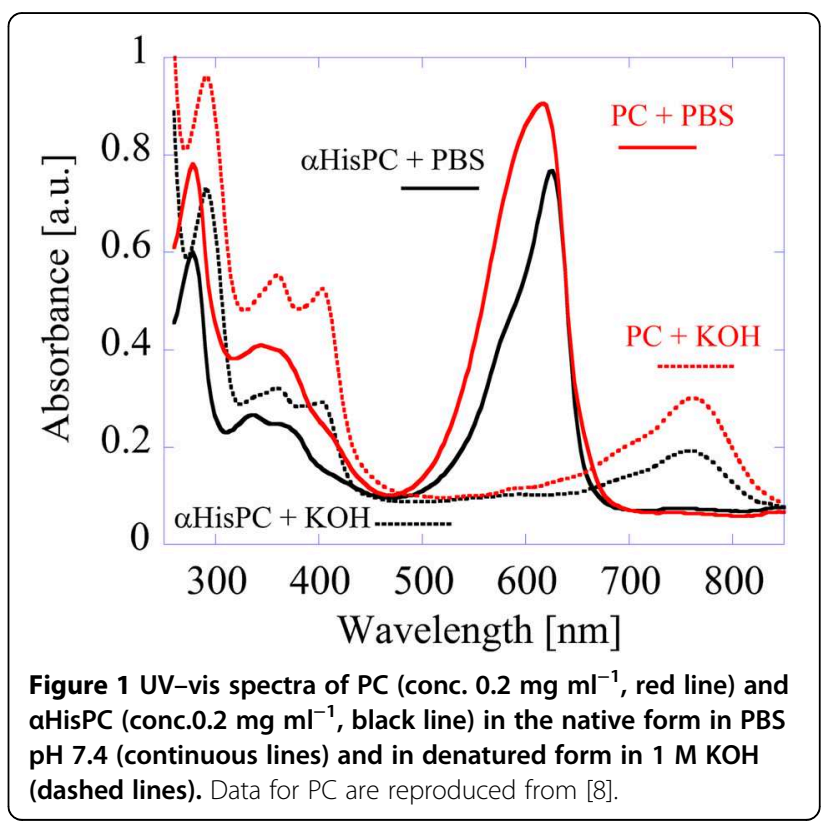


3.2 Effect of protein-melanin coating on the photocurrent In order to establish optimal parameters for the coating process, three protein concentrations spanning two orders of magnitude $\left(0.02,0.2\right.$ and $2 \mathrm{mg} \mathrm{ml}^{-1}$ ) and three reaction times $(1.5,3$ and $4.5 \mathrm{~h})$ are tested. The highest enhancement in photocurrent density was achieved at concentrations between $0.2 \mathrm{mg} \mathrm{ml}^{-1}$ to $2 \mathrm{mg} \mathrm{ml}^{-1}$ of PC and a reaction time of $3 \mathrm{~h}$ to $4.5 \mathrm{~h}$. For hematite thin films coated with $\mathrm{PC}$, the highest relative enhancement in photocurrent density is $23 \%$ and is obtained at a concentration of $2 \mathrm{mg} \mathrm{ml}^{-1}$ and a reaction time of $3 \mathrm{~h}$, when measured in $\mathrm{KOH}$. Under identical conditions, a higher value is obtained with samples coated with $\alpha \mathrm{HisPC}$ at a concentration of $0.2 \mathrm{mg} \mathrm{ml}^{-1}$ that give a $55 \%$ increase in photocurrent density.

Figure 2 shows a typical I-V (photocurrent density) curve of the pristine and the protein coated electrodes in $\mathrm{KOH}$ (Figure 2a) and PBS (Figure 2b) electrolytes. The protein layer causes the higher photocurrent density. The higher photocurrent densities can be attributed to the panchromatic light harvesting action of protein and the electronic transfer to the hematite surface. The photocurrent onset potential for both samples is virtually the same vs. $\mathrm{Ag} / \mathrm{AgCl}$, i.e. $\sim 0 \mathrm{~V}$ in $\mathrm{KOH}$ and $600 \mathrm{mV}$ in PBS, when we account for the effect of the different $\mathrm{pH}$ on the Nernst potential.

We expect that the extreme $\mathrm{pH}$ conditions with the use of $1 \mathrm{M} \mathrm{KOH}$ electrolyte ( pH 13-14) leads to melanin dissolution and structural changes in PC causing denaturation. Yet we measure in $\mathrm{KOH}$ a significant increase in photocurrent density (left panel, Figure 2a). A possible explanation might be found in the newly occurring absorbance feature at $750 \mathrm{~nm}$ for PC and $\alpha \mathrm{HisPC}$, when measured in the strongly alkaline environment (Figure 1). While the proteins may become denatured, the chromophores may still be intact and absorb light [10] and produce electron-hole pairs which add to the photocurrent. Moreover, the recombinant monomeric $\alpha$ HisPC appears to be a valid alternative to the commercial PC preparation. The 55\% increase measured for $\alpha$ HisPC is significantly higher than the $8 \%$ increase measured for analogous samples prepared with PC (all measured in $1 \mathrm{M} \mathrm{KOH})$. We thus speculate that $\mathrm{PC}$ and $\alpha$ HisPC couple differently with the surface, thus conferring different charge transfer. As a possible explanation, the presence of the Histag on $\alpha$ HisPC might provide a better conducting bonding to the hematite surface as histidine residues are known to coordinate iron ions and the Histag has been shown to bind iron in some proteins [11]. For the evaluation of the effect of melanin alone, samples lacking any photosynthetic protein were prepared and analysed as a control experiment. Compared to the hematite films with coatings containing $\mathrm{PC}$ or $\alpha$ HisPC and melanin, no significant increase in photocurrent was observed. Therefore the key factor for the increase in photocurrent is the biliprotein, and not the melanin. Based on these results, a $0.2 \mathrm{mg} \mathrm{ml}^{-1}$ protein concentration and $3 \mathrm{~h}$ enzymatic reaction time was used for further experiments.

\subsection{Surface morphology of phycocyanin-melanin coated samples}

Electron microscopy was used for the closer visual inspection of the hematite coated with melanin and different amounts of PC. Electron micrographs of hematite coated with melanin and $\mathrm{PC}$ show the formation of strands of protein-melanin complexes (Figure 3). Since the same concentration of melanin was present in all samples, strand formation depends on the protein concentration. At the lower $0.2 \mathrm{mg} \mathrm{ml}^{-1}$ protein concentration, SEM micrographs of samples coated with PC (Figure 3) shows formation of stretched dense formations in tight contact with the hematite surface, and this could play a role in the enhancement of the photocurrent. A significant strand formation is visible in samples with a PC concentration of $2 \mathrm{mg} \mathrm{ml}^{-1}$ rather than at
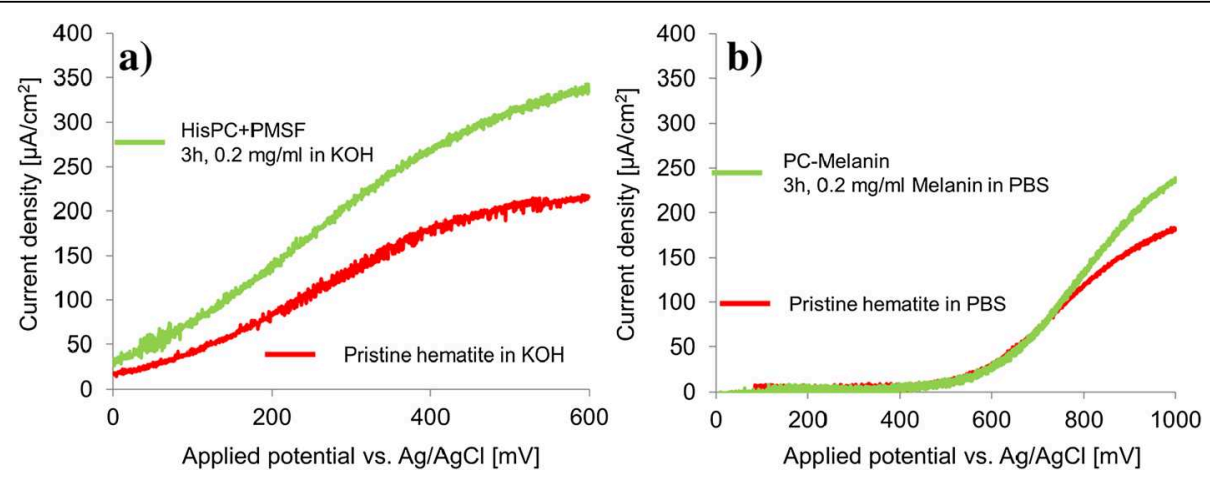

Figure 2 Photocurrent density before and after immobilization of aHisPC (a) and PC (b). Pristine hematite before (red bottom line) and after immobilization with melanin (green top line) of $0.2 \mathrm{mg} \mathrm{ml}^{-1} \mathrm{PC}$ or aHisPC in the presence of $1 \mathrm{mM} \mathrm{PMSF}$, for $3 \mathrm{~h}$ and measured in $1 \mathrm{M} \mathrm{KOH}$ (left panel) and in 0.05 M PBS (right panel). 

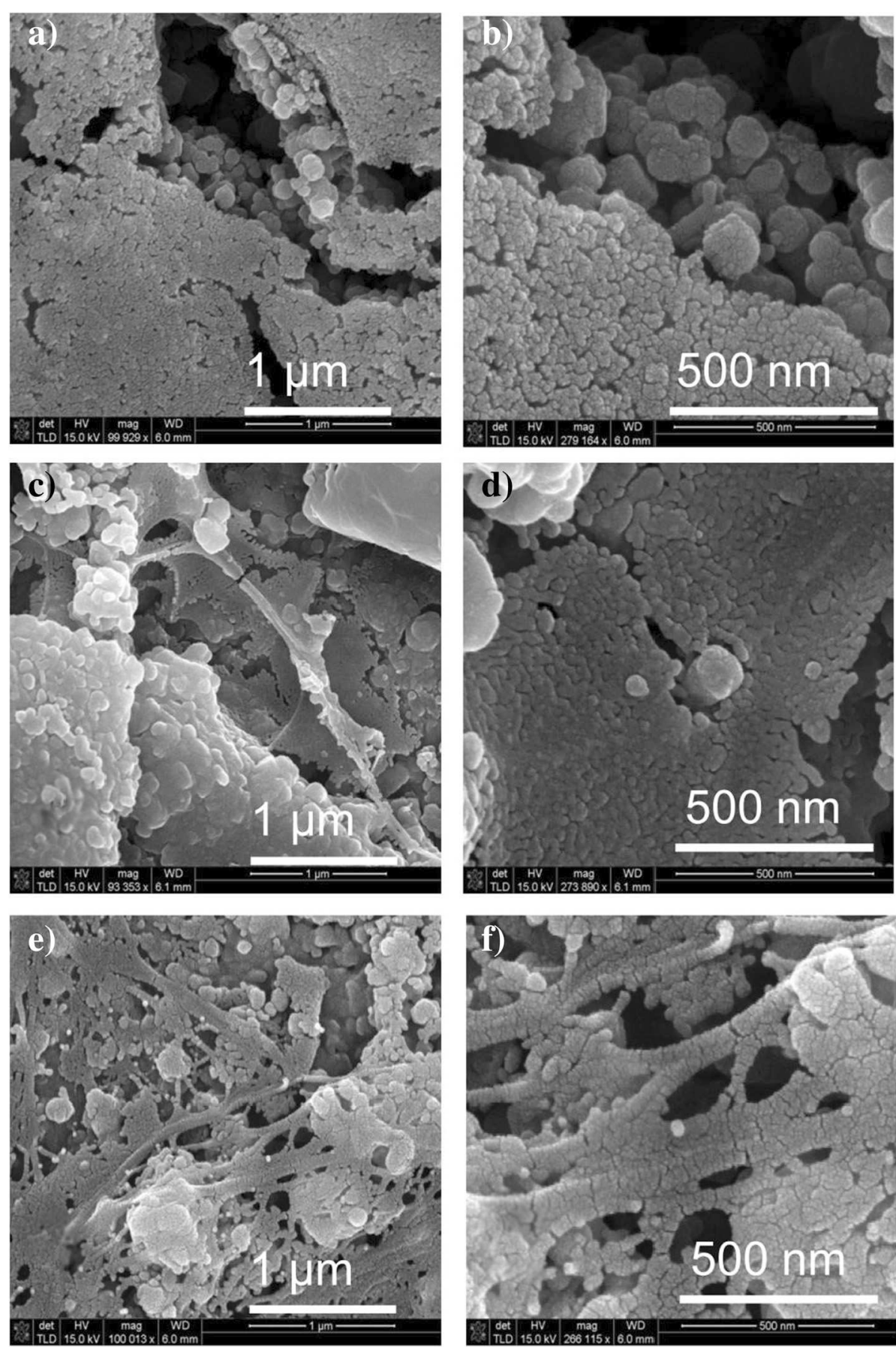

Figure 3 Scanning electron micrographs of hematite films coated with different amounts of PC, e.g. incubation for $3 \mathrm{~h}$ with a melaninforming mixture containing $0.02(a, b), 0.2(c, d)$ and $\mathbf{2}(e, f) \mathbf{~ m g ~ m l}^{-1}$ incubated from top to bottom row. The scale bar is $1 \mu \mathrm{m}$ (left) or $0.5 \mu \mathrm{m}$ (right).

$0.2 \mathrm{mg} \mathrm{ml}^{-1}$. In addition to the protein strains, spherical granules with a diameter of $200 \mathrm{~nm}$ are observed (Figure 3). These are likely due to melanin which tends to organise in such granules when isolated from natural sources [12]. The pattern observed differs from that reported by Costa et al. [13] who studied natural and synthetic melanin and their interaction with Fe (III) by SEM. However, such heterogeneous kind of geometry was also described in [14] using laser light scattering which also showed how enzymatically-produced melanin can be largely hydrated and can have non-uniform density. However, the highest enhancement in current density 
was measured for samples with the $0.2 \mathrm{mg} / \mathrm{ml} \mathrm{PC}$ concentration.

\subsection{Charge transfer in PC-melanin coated hematite films} Important for the functionality of bio-inorganic interfaces in sensors or energy converters is not only their physical immobilization but also sufficient charge transfer between the organic component and the inorganic component, i.e. the electrode of the current collector. Charge transfer between electrodes and proteins or even whole cells has been studied for various conducting substrates, for example for GaAs and photosystem I [15], gold and blue copper protein azurin [16], carbon nanotubes [17] and for stainless steel [18]. It is clear from numerous examples, that electrons can be directly transferred from and to proteins which are in contact with inorganic surfaces, including hematite. Impedance spectroscopy can be a valuable analytical tool for the assessment of bio-organic interfaces [19]. Dheilly et al. [20] used electrochemical impedance spectroscopy (EIS) to probe the dynamics of the charge transfer of bacterial biofilms attached to electrodes in an aqueous environment. We apply EIS for studying the interaction between the photosensitizing protein (PC) and hematite using different coating procedures under dark and illuminated conditions using PBS as electrolyte. For the reader we illustrate in Figure 4 the sequence of the various synthesis and coating steps for the assembly of the electrode.
The schematic bio-hybrid electrode architecture in Figure 5a) shows one possible impedance model circuit for our protein-semiconductor assembly (following a suggestion of Ben-Yoav et al. [22]), along with an energy diagram for the solid state processes and the processes in the electrolyte. The water splitting reaction is based on electron holes $\mathrm{h}^{+}$which come from the hematite photoanode: $2 \mathrm{H}_{2} \mathrm{O}+4 \mathrm{~h}^{+} \rightarrow \mathrm{O}_{2} \uparrow+4 \mathrm{H}^{+}$. The excited PC molecule generates also an electron-hole pair. The electron moves from the PC to the hematite. Since the highest occupied molecule orbital (HOMO) energy level of $\mathrm{PC}$ is above the VB of hematite, the electron hole cannot transfer from $\mathrm{PC}$ to hematite. Rather, the hole $\mathrm{h}^{+}$from the PC can form with the aqueous electrolyte hydroxyl radicals $\left({ }^{\bullet} \mathrm{HO}\right)$ upon excitation with visible light $>470 \mathrm{~nm}$ wavelength [23]. This would balance the charge transfer via the Type I mechanism [24]. This constitutes an electron acceptor. Backflow of electrons to the electrolyte via the surface would be prevented by the ${ }^{\bullet} \mathrm{HO}$ radicals. Since the electron does not get freedom to recombine with holes at the hematite surface and back injected to electrolyte, the functionalization with $\mathrm{PC}$ increases the photocurrent density.

Figure 6 shows the impedance spectra of the pristine hematite film coated on FTO glass, measured in PBS in the dark (Figure 6a) and under 1.5 AM solar simulated light (Figure 6b). The spectra were recorded with bias ranging from $0 \mathrm{mV}$ to $1000 \mathrm{mV}$ in steps of $200 \mathrm{mV}$ at

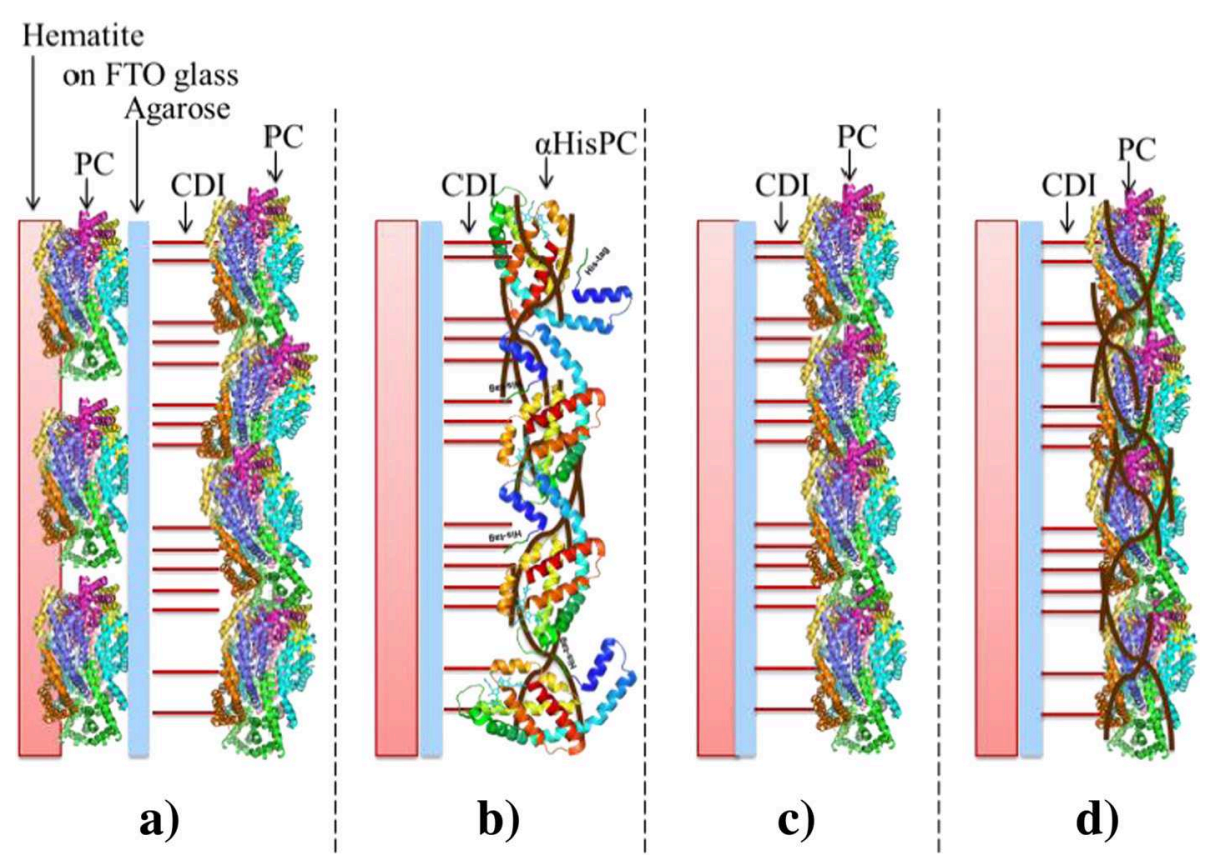

Figure 4 Schematic of the applied coating procedures for PC and aHisPC covalently attached to hematite via a chemical crosslinker, e.g. CDI. Differently from Bora [21], as shown here for reference in a), the hematite surface is directly coated with agarose and melanin using the chemical crosslinker CDI. Distances are not in proportion. Additionally, a layer containing melanin and aHisPC (b, upper panel, PDB ID: 4F0T), PC (c upper panel), or PC plus melanin (d upper panel, PDB ID: 1HA7) was applied. 

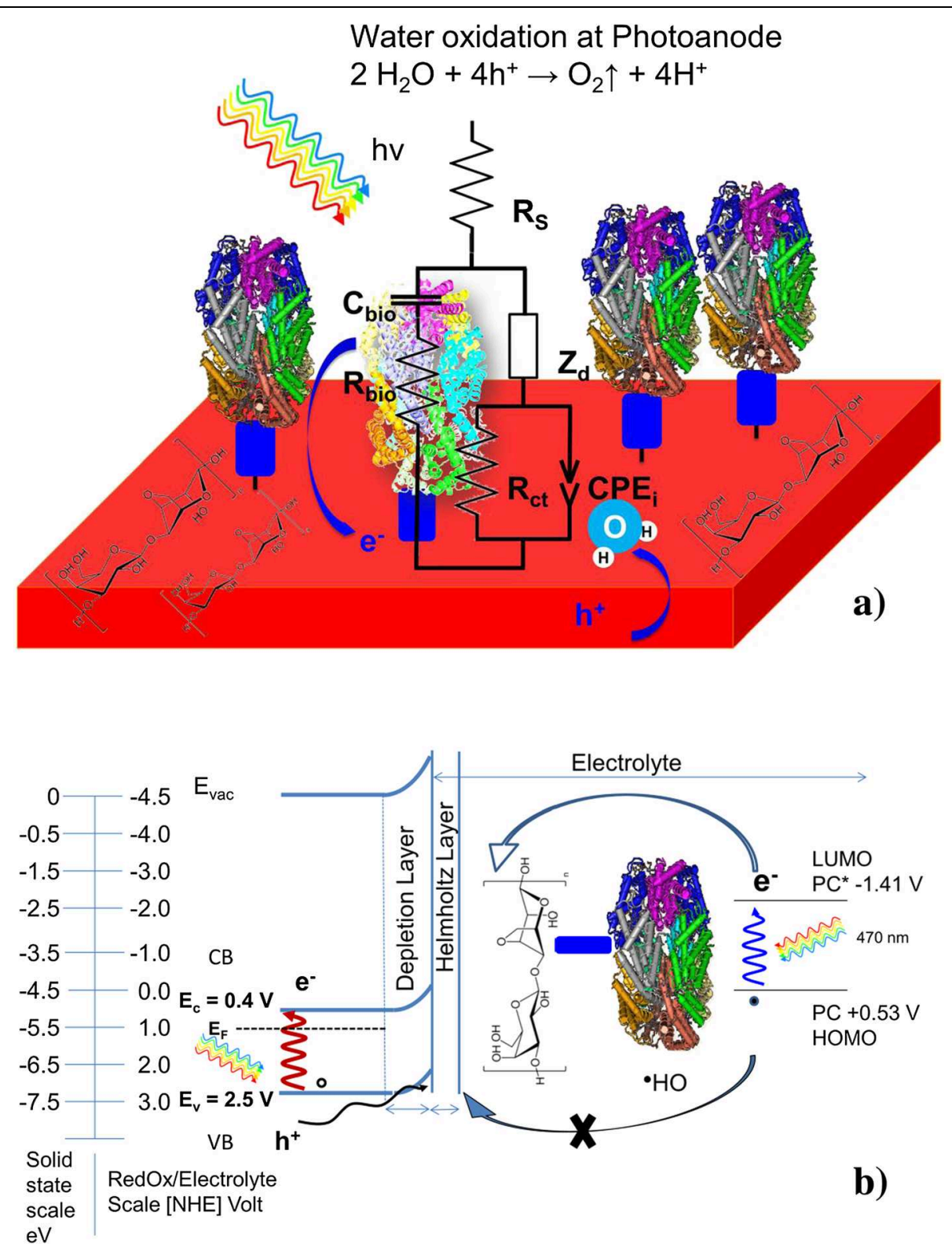

Figure 5 Top a) - The semiconducting hematite interfacing the electrolyte is represented by the charge transfer resistance $R_{c t}$ and a constant phase element $C P E_{i}$ and a Warburg impedance element $Z_{d} \cdot R_{s}$ is the resistance of the electrolyte. Inspired by reference [22]. Bottom $\mathbf{b})$ - Energy diagram for the PC in aqueous electrolyte, linked with CDI and agarose to hematite.

frequencies ranging from $100 \mathrm{kHz}$ (low impedance point near origin) to $0.1 \mathrm{~Hz}$ (high impedance point at end of spectrum). The impedance spectra of pristine hematite in dark fit in the impedance window posed by axes with $10 \mathrm{kOhm}$. Under illumination, the spectra obtained under $600 \mathrm{mV}$ to $1000 \mathrm{mV}$ bias show a convolution of two semicircles (Figure 6b) for the spectrum obtained at $800 \mathrm{mV}$. The first semicircle has a significantly smaller radius than the second one. The radius of the larger of both semicircles scales systematically with the applied DC bias and thus represents the charge transfer resistance.
For the hematite film coated with protein, the impedance measured in dark condition is below $5 \mathrm{kOhm}$ (Figure 7a), revealing that the protein layer ( $\alpha$ HisPC coated with the enzymatic melanin formation procedure) improves charge transfer between electrolyte and hematite. Note that we have plotted the data in Figures 6 and 7 in the same scaled window for easier comparison.

Moreover, upon illumination of the protein film, the impedance decreases dramatically (see the very small box in the lower left corner in the impedance spectrum in $7 \mathrm{~b})$ to far below $1 \mathrm{kOhm}$. Here it is sufficient to plot the impedance in an isometric window of $300 \mathrm{Ohm}$ to 

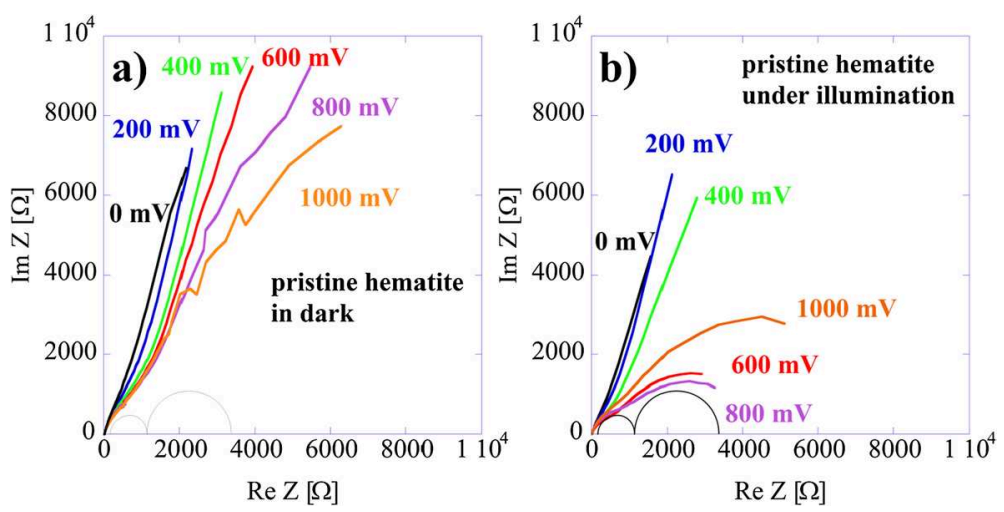

Figure 6 EIS spectra (Nyquist plots) of pristine hematite in dark a) and under illumination b) for DC bias from $0 \mathrm{mV}$ to $1000 \mathrm{mV}$. The spectra are plotted on the same isometric scale.

present virtually the entire impedance spectra when measured under light condition. The lowest impedance is found for $800 \mathrm{mV}$ bias. The curvature of the spectra in the inset of Figure 7b) shows the clear tendency towards semicircles as the direct current (DC) bias approaches the water splitting potential. We interpret this as increased charge transfer of the system during illumination and increased DC bias. The protein coating improves the conductivity and charge transfer between the hematite and the electrolyte also under illumination significantly. The impedance for pristine and protein coated hematite increases again in the transition from $800 \mathrm{mV}$ to $1000 \mathrm{mV}$. This is, where typically considerable oxygen evolution by water oxidation takes place.

Charge transfer across interfaces depends on the electronic structure of these interfaces. A method for the assessment of the electronic structure of surfaces and interfaces is X-ray and photoelectron spectroscopy (XPS) and photoemission spectroscopy (PES). In Brizzolara et al. [25] the conclusion about covalent attachment of the proton pump protein bacteriorhodopsin via genetic substitution of cysteine for serine (S35C) was based partially on the XPS core level spectra of sulfur, i.e. via detection of the chemical shift of the sulphur core level spectrum. An early valence band (VB) XPS study on a protein (D-luciferin) is presented in Wada et al. [26], where XPS helped sketch a model for the luminescence. In this study, we find that the position of the VB spectrum changes depending on the type of phycocyanin coating process applied to hematite.

Figure 8 shows a set of VB spectra which were recorded with photoemission spectroscopy (PES) with photon energies close to the Fe $3 p$ resonance edge, $\sim 54 \mathrm{eV}$. The PES was employed in resonance so as to identify the contribution of the Fe ions in the VB density of states (DOS). The spectra were recorded from three different samples, Figure 8a) PC coated on hematite single crystal, Figure $8 \mathrm{~b}$ ) $\mathrm{PC}$ and melanin $(\mathrm{PC}+\mathrm{Mel})$ co-polymerized on hematite single crystal, and Figure 8c) His-tagged PC and melanin $(\alpha \mathrm{HisPC}+\mathrm{Mel})$ on hematite single crystal.

We observe that, under dark condition (8a), the intensity maximum of the spectrum from the PC film shifts
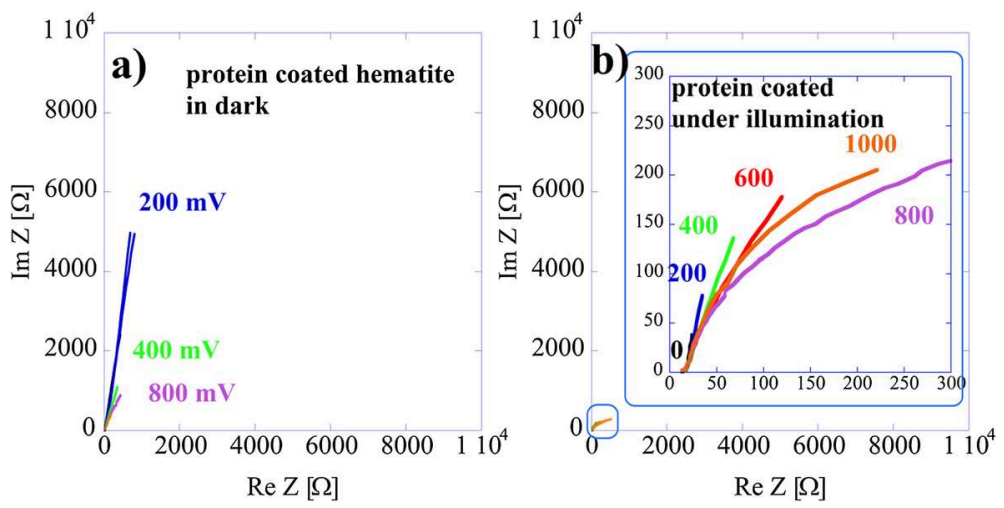

Figure 7 EIS spectra (Nyquist plots) of protein coated hematite recorded in dark a) and under illumination b) for DC bias from $0 \mathrm{mV}$ to $1000 \mathrm{mV}$. The inset on the right shows the largest magnification. Note the very small window in the lower left corner, showing the very small overall impedance of the illuminated protein film. Coating procedure: enzymatic, aHisPC plus melanin (condition B in Figure 4, upper panel) [8]. 


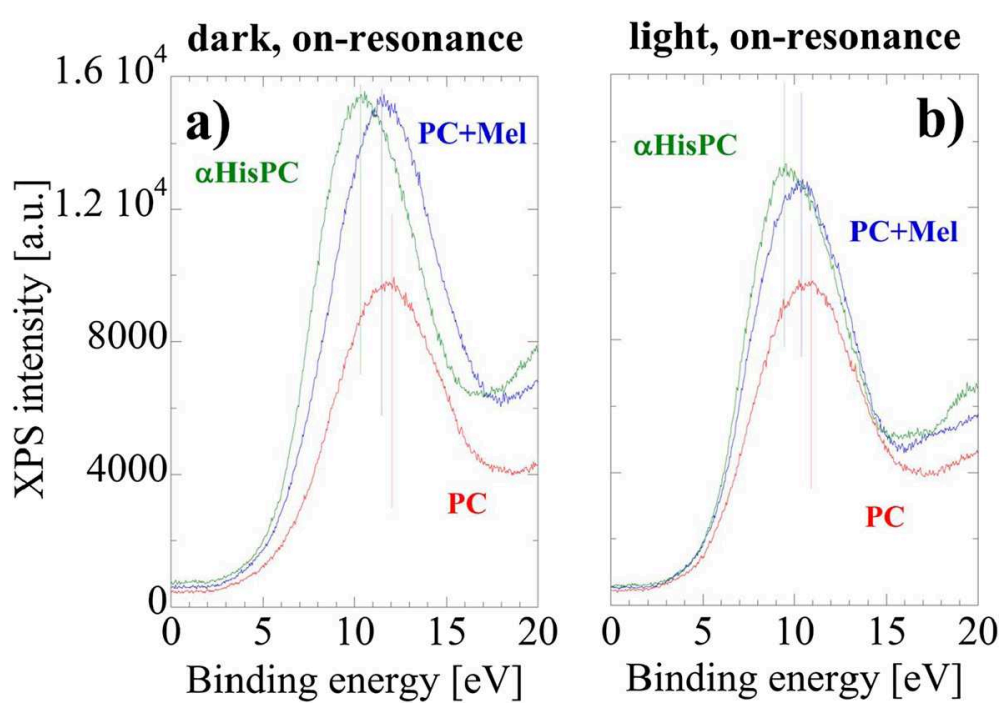

Figure 8 Comparison of three different coatings (condition B-D in Figure 4, upper panel) on hematite in Fe3p on-resonance condition at $\mathbf{5 4 . 4} \mathrm{eV}$ photon energy under dark (a) and illuminated (b) conditions. With more advanced film processing technology, the VB PES spectra shifts closer towards the Fermi level (0 eV binding energy).

from $12 \mathrm{eV}$ to just below $11.5 \mathrm{eV}$ towards the Fermi level, when PC was co-polymerized with melanin (Figure 8a). With $\alpha \mathrm{HisPC}+\mathrm{Mel}$ on hematite, the intensity maximum shifts to $10.3 \mathrm{eV}$. A similar trend is shown in the case of illumination with the solar simulator (Figure $8 \mathrm{~b}$ ). We notice that with increasing improvement of film processing technology, the maxima of the VB spectra move closer to the Fermi level $E_{F}$ reflecting the changes in the electronic structure with increased DOS near $\mathrm{E}_{\mathrm{F}}$. The charge transfer between hematite and phycocyanin might be improved in $\alpha$ HisPC as histidines are known to coordinate to iron ions. At this time it is not clear whether the spectral shift towards the $E_{F}$ is a result of hole doping originating from the $\mathrm{PC}+\mathrm{Mel}$ in the films. In addition, the His-tag presumably improves entrapment of the His-tagged protein into the melanin network, as the formation of histidinetyrosine bonds have been observed upon tyrosinasecatalysed crosslinking [27].

It is clear, however, that there is a correlation between improved electric transport, increased photocurrent and increased DOS in the $\mathrm{VB}$ near $\mathrm{E}_{\mathrm{F}}$. Although literature on the electronic structure of the interfaces of biological macromolecules and inorganic materials is scarce, some pioneering studies have been published. A PES and NEXAFS study on the electronic structure of an ultrathin film of the surface layer of Bacillus sphaericus deposited on Si wafers showed how the highest occupied and lowest unoccupied molecular orbitals (HOMO and LUMO) constitute the band gap of the assembly [28]. In an extension of that study, these authors showed with resonant PES how charge transport evolved from torsion effects in the protein [29].
We want to turn to another observation in our PES spectra. When subjecting the protein-hematite assembly to $1.5 \mathrm{AM}$ simulated solar light, the spectral weight of the intensity range near the $\mathrm{O} 2 \mathrm{p}$ bonding peak decreases and shifts by up to $0.7 \mathrm{eV}$ toward the Fermi level $(0 \mathrm{eV}$ binding energy).

We interpret this shift as the hole-doped DOS resultant from the exposure of visible light. With the solar lighting on, the electron-hole pairs can be created in the conduction band (CB) and the holes stays near the surface generating a p-type DOS. A similar trend of binding energy shift is observed when silicon is doped [30]. Our PES studies on proteins are in principal not different from studies on synthetic organic molecules and polymers which are frequently used in solar cells and light emitting diodes. A comprehensive review on such VB spectroscopy studies is presented by Koch et al. [31].

Among our protein functionalization processes, the coating with PC only (Figure 9a) is the least complex one. For the comparison of the PES spectra in Figure 9, we determine the position of the VB by extrapolation of the spectral flank near $E_{F}$ towards zero intensity - as a simple and practical metric. The intercept is then by our definition the VB position. The VB position of the PES spectra shifts from $6 \mathrm{eV}$ to $5 \mathrm{eV}$ when the sample is illuminated with the solar simulator (Figure 9a). This $1 \mathrm{eV}$ shift thus originates from the illumination effect, i.e. a charge carrier (electron hole pair) generation.

When we turn to the PC + Mel coated hematite (Figure 9b), the $\mathrm{VB}$ position shifts from $5.6 \mathrm{eV}$ to $5 \mathrm{eV}$, i.e. the shift is only $0.6 \mathrm{eV}$ upon illumination. In the case of the HisPC coating (Figure 9c), there is no shift of the VB spectrum 


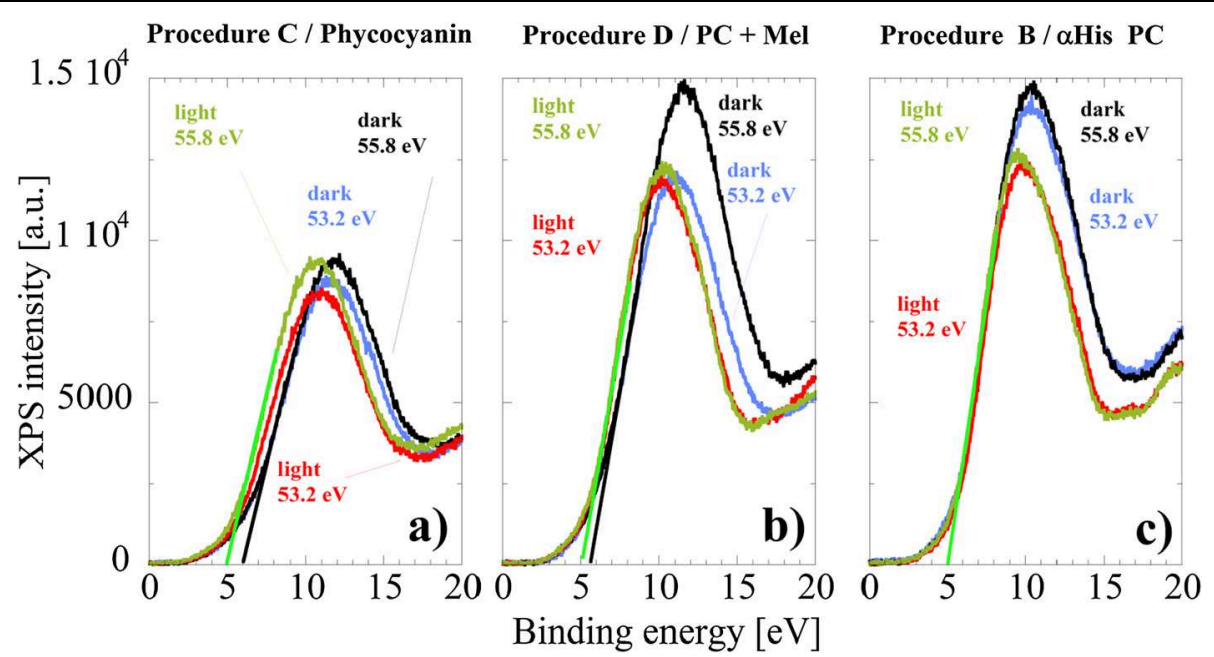

Figure 9 Comparison of VB PES spectra for the three different protein coatings (procedures C, D, and B from left to right from Figure 4). The steep straight lines intersecting near $5 \mathrm{eV}$ show the VB position. The sample coated with PC and melanin ( panel b), procedure D in Figure 4) shows $0.6 \mathrm{eV}$ shift on VB position while PC only ( panel a), procedure $C$ in Figure 4) shows $1.0 \mathrm{eV}$ shift under illumination. In the case of aHisPC ( panel c) ), no shift in the VB position is observed.

when the light is switched on. Interestingly, for all films, the position of the $\mathrm{VB}$ in the illumination condition is $5 \mathrm{eV}$. Hence, it appears that light has no influence on the charge transfer as far as the interface of the protein film with the hematite is concerned.

In addition to the electric charge transfer, which is the scope of this paper, fluorescence resonance energy transfer (FRET) could hypothetically occur between phycocyanin and hematite and thus increase the photocurrent density. The efficiency of the FRET mechanism is inversely proportional to the sixth power of the distance between light donor and light acceptor, making it extremely sensitive to distances and virtually negligible for small distances. Due to the architectures of our biohybrid electrodes, where agarose, CDI and His tag virtually constitute "spacers" between PC and hematite, it is difficult to perceive that FRET could occur across these spacers.

\section{Conclusions}

The immobilization of photosynthetic proteins on hematite, in particular C-phycocyanin, yields a substantial increase of the photocurrent density which can be assigned to electron hole pair formation in the protein by light absorption in a wavelength range that exceeds the absorption range of the hematite. Refined immobilization strategies like copolymerization of $\mathrm{PC}$ with melanin or genetic engineering to attach a His-Tag to PC successively increase the photocurrent. Analysis of the charge carrier dynamics of the bio-hybrid electrode assembly under photoelectrochemical conditions shows that upon illumination the enhanced charge transfer and DC bias are substantially amplified (factor 10) when the hematite film is coated with the light harvesting protein. This, however, seems to have not the same strong effect on the photocurrent density (only factor 2). A clear and systematic dependency of spectral shift of the valence band is observed in the dark and in the illuminated condition. While the His-tag strategy clearly improves the charge transfer between $\mathrm{PC}$ and hematite in the dark, no further enhancement is observed under illumination condition. This might be due to the fact that the illumination generates electron hole pairs, but this should have no effect on the charge transport characteristic of the covalent linkages between protein and hematite.

\section{Competing interests}

The authors declare that they have no competing interests.

\section{Authors' contributions}

GF synthesized and processed samples and wrote major part of the manuscript. KGS synthesized and processed samples and measured current densities. $J$ organized the work, inspired the use of melanin and provided intellectual input on protein functionalization. FB measured the protein samples with photoemission spectroscopy at SSRL and ALS. YH measured the protein samples with photoemission spectroscopy at SSRL and ALS and analyzed the impedance spectra and arranged for SSRL beamtime. BSM measured the protein samples with photoemission spectroscopy at SSRL and ALS and discussed the spectra. DKB provided intellectual input in. LTM organized the work and provided intellectual input. $A B$ organized the work, inspired the protein functionalization, provided intellectual input, did data analysis on photoemission spectra, arranged for ALS beamtime and wrote major part of the manuscript. All authors read and approved the final manuscript.

\section{Acknowledgements}

This study was funded at large by the VELUX Foundation under project no ${ }^{\circ}$ 790 (Biomimetic photoelectrochemical cells for solar hydrogen generation: Bio-PEC) for K. G.-S. and G.F. G.F. was also financially supported by the TYROMAT project (COFUND action between Empa Postdocs programme and FP7: People) and COST TD1102 Action Project by SBFI C13.003. Financial support by the Strategic Korean-Swiss Cooperative Program in Science and 
Technology within the project "Spectroscopy on photoelectrochemical electrode materials: SOPEM" for A.B. and NRF-2013K1A3A1A14055158 for B.S.M. is gratefully acknowledged. Financial support by the Swiss National Science Foundation for F.B. (project \# 200021-137868 Reaction-diffusion processes for the growth of patterned structures and architectures: bottom up approach for photoelectrochemical electrodes) and for Y.H. (project \# 200021-132126 Defects in the bulk and on surfaces and interfaces of metal oxides with photoelectrochemical properties: In-situ photo-electro-chemical and resonant x-ray and electron spectroscopy studies) and R'Equip \# 121306 is gratefully acknowledged.

Portions of this research were carried out at the Stanford Synchrotron Radiation Lightsource, a national user facility operated by Stanford University on behalf of the U.S. Department of Energy, Office of Basic Energy Sciences. The solar simulator was provided by Jinghua Guo, Advanced Light Source in Berkeley. Florian Häussler (Empa Dübendorf) assisted with the sample preparation at Empa. We are grateful to Prof. Wendy Schluchter from the Department of Biological Sciences of the University of New Orleans for providing the plasmids for the production of aHisPC in this study.

\section{Author details}

'Laboratory for Bioactive Materials, Empa, Swiss Federal Laboratories for Materials Science and Technology, Lerchenfeldstrasse 5, 9014 St. Gallen, Switzerland. 'Laboratory for High Performance Ceramics, Empa, Swiss Federal Laboratories for Materials Science and Technology, Überlandstrasse 129, 8600 Dübendorf, Switzerland. ${ }^{3}$ Department of Inorganic and Analytical Chemistry, Szeged University, Dóm tér 7, H-6720 Szeged, Hungary. ${ }^{4}$ Institute of Chemistry, Research Group of Environmental Chemistry, University of Szeged, Rerrich Béla tér 1, H-6720 Szeged, Hungary. ${ }^{5}$ Department of Chemistry, University of Basel, Spitalstrasse 51, 4056 Basel, Switzerland. ' ${ }^{6}$ aboratory for Photonics and Interfaces, Ecole Polytechnique Federale de Lausanne, Rue Cantonale, 1021 Lausanne, Switzerland. 'Department of Physics and Photon Science, GIST School of Physics and Chemistry, GIST College, Ertl Center for Electrochemistry and Catalysis, 123 Cheomdan-gwagiro (Oryong-dong), Buk-gu, Gwangju 500-712, Korea.

Received: 2 November 2014 Accepted: 7 November 2014

Published online: 06 May 2015

\section{References}

1. Bora DK, Braun A, Constable EC: Energy Environ. Sci. 2013, 6(2):407-425.

2. Anderson S, Constable EC, Dare Edwards MP, Goodenough JB, Hamnett A, Seddon KR, Wright RD: Nature 1979, 280(5723):571-573.

3. Tooley AJ, Cai YP, Glazer AN: Proc. Natl. Acad. Sci. 2001, 98(19):10560-10565.

4. Fairhead M, Thoeny-Meyer L: New Biotechnol 2012, 29(2):183-191.

5. Taurino I, Reiss R, Richter M, Fairhead M, Thoeny-Meyer L, De Micheli G, Carrara S: Electrochim. Acta. 2013, 93:72-79.

6. Hu Y, Bora DK, Boudoire F, Haeussler F, Graetzel M, Constable EC, Braun A: J. Renew. Sust. Ener. 2013, 5(4):043109.

7. Chen Z, Jaramillo TF, Deutsch TG, Kleiman-Shwarsctein A, Forman AJ, Gaillard N, Garland R, Takanabe K, Heske C, Sunkara M, McFarland EW, Domen K, Miller EL, Turner JA, Dinh HN: J. Mater. Res. 2010, 25(1):3-16.

8. Ihssen J, Braun A, Faccio G, Gajda-Schrantz K, Thöny-Meyer L: Light harvesting proteins for solar fuel generation in bioengineered photoelectrochemical cells. Curr. Protein. Pept. Sci. 2014, 15: in press.

9. Glazer AN, Fang S, Brown DM: J. Biol. Chem. 1973, 248:5679-5685.

10. Berns DS, MacColl R: Chem. Rev. 1989, 89:807-825.

11. The Recombinant Protein Handbook: Protein amplification and simple purification. Amersham Biosciences 2002, 41-58. 18-1142-75.

12. Watt AA, Bothma JP, Meredith P: Soft Matter 2009, 5(19):3754-3760.

13. Costa TG, Younger R, Poe C, Farmer PJ, Szpoganicz B: Bioinorg. Chem. Appl. 2012, 2012:712840.

14. Bridelli MG: Biophys. Chem. 1998, 73(3):227-239.

15. Frolov L, Rosenwaks Y, Richter S, Carmeli C, Carmeli I, Phys J: Chem. C. 2008, 112:13426-13430.

16. Chi Q, Farver O, Ulstrup J: PNAS 2005, 102(45):16203-16208.

17. Deng S, Jian G, Lei J, Hu Z, Ju H: Biosens Bioelectron 2009, 25:373-377.

18. Omanovic S, Roscoe SG: Langmuir 1999, 15(23):8315-8321.

19. Owino IOK, Sadik OA: Electroanalysis 2005, 17(23):2101-2113.

20. Dheilly A, Linossier I, Darchen A, Hadjiev D, Corbel C, Alonso V: Appl. Microbiol. Biotechnol. 2008, 79(1):157-164.
21. Bora DK, Rozhkova AA, Schrantz K, Wyss PP, Braun A, Graule T, Constable EC: Adv. Funct. Mater. 2012, 22(3):490-502.

22. Ben-Yoav H, Freeman A, Sternheim M, Shacham-Diamand Y: Electrochim. Acta. 2011, 56(23):7780-7786.

23. Zhang S, Xie J, Zhang J, Zhao J, Jiang L: Biochim. Biophys. Acta. 1999, 1426(1):205-211.

24. Foote CS: Photochem. Photobiol. 1991, 54(5):659.

25. Brizzolara RA, Boyd JL, Tate AE, Vac J: Sci. Technol. A. 1997, 15(3):773-778.

26. Wada N, Shitaba R, Shibazaki M, Suzuki N: Photochem. Photobiol. 1989, 49(4):513-518

27. Hellman M, Mattinen M-L, Fu B, Buchert J, Permi P: J. Biotechnol. 2011, 151(1):143-150.

28. Vyalikh DV, Danzenbacher S, Mertig M, Kirchner A, Pompe W, Dedkov YS: Phys. Rev. Lett. 2004, 93(23):238103.

29. Vyalikh DV, Maslyuk W, Blueher A, Kade A, Kummer K, Dedkov YS: Phys. Rev. Lett. 2009, 102(9):098101.

30. Sezen H, Suzer S: J. Chem. Phys. 2011, 135:141102.

31. Koch N: phys. stat. sol. RRL. 2012, 6(7):277-293.

\section{Submit your manuscript to a SpringerOpen ${ }^{\circ}$ journal and benefit from:}

- Convenient online submission

- Rigorous peer review

- Immediate publication on acceptance

- Open access: articles freely available online

- High visibility within the field

- Retaining the copyright to your article

Submit your next manuscript at $>$ springeropen.com 